\title{
Angiotensin II receptor 1 gene variants are associated with high-altitude pulmonary edema risk
}

\author{
Tianbo Jin ${ }^{1,2,3,4, *}$, Yongchao Ren ${ }^{4,5, *}$, Xikai Zhu ${ }^{1,2,3}$, Xun Li ${ }^{6}$, Yongri Ouyang ${ }^{4}$, Xue \\ He $^{1,2,3}$, Zhiying Zhang ${ }^{1,2,3}$, Yuan Zhang ${ }^{1,2,3}$, Longli Kang ${ }^{1,2,3}$, Dongya Yuan ${ }^{1,2,3}$ \\ ${ }^{1}$ Key Laboratory for Molecular Genetic Mechanisms and Intervention Research on High Altitude disease of Tibet Autonomous \\ Region, School of Medicine, Xizang Minzu University, Xianyang, Shaanxi 712082, China \\ ${ }^{2}$ Key Laboratory of High Altitude Environment and Genes Related to Diseases of Tibet Autonomous Region, School of \\ Medicine, Xizang Minzu University, Xianyang 712082, China \\ ${ }^{3}$ Key Laboratory for Basic Life Science Research of Tibet Autonomous Region, School of Medicine, Xizang Minzu University, \\ Xianyang, Shaanxi 712082, China \\ ${ }^{4}$ School of Life Sciences, Northwest University, Xi'an, Shaanxi 710069, China \\ ${ }^{5}$ Qiannan Institute for Food and Drug Control, Duyun, Guizhou 558000, China \\ ${ }^{6}$ The Center of Altitude Disease, General Hospital of Tibet Military Area Command, Lasa 850000, China \\ *These authors have contributed equally to this work \\ Correspondence to: Dongya Yuan, email: yuandongyaXz@163.com \\ Longli Kang, email: longlikang@ 126.com
}

Key words: high-altitude pulmonary edema (HAPE), AGTR 1, polymorphisms, haplotype

Received: June 07, $2016 \quad$ Accepted: September 25, $2016 \quad$ Published: October 06, 2016

\section{ABSTRACT}

Previous studies demonstrated that Angiotensin II Receptor 1 (AGTR1) may play an important role in the development of high-altitude pulmonary edema. We envisaged a role for AGTR1 gene variants in the pathogenesis of HAPE and investigated their potential associations with HAPE in a Han Chinese population. We genotyped seven AGTR1 polymorphisms in 267 patients with diagnosed HAPE and 304 controls and evaluated their association with risk of HAPE. Statistically significant associations were found for the single nucleotide polymorphisms (SNPs) rs275651 $(p=0.017$; odds ratio $[O R]=0.65)$ and $\operatorname{rs} 275652(p=0.016 ;$ OR $=0.64)$. Another SNP rs10941679 showed a marginally significant association after adjusting for age and sex in the additive genetic model (adjusted OR $=1.44,95 \% \mathrm{CI}=1.01-2.04, p=$ 0.040). Haplotype analysis confirmed that the haplotype "AG" was associated with a $35 \%$ reduction in the risk of developing HAPE, while the haplotype "AA" increased the risk of developing HAPE by $44 \%$. These results provide the first evidence linking genetic variations in AGTR1 with HAPE risk in Han Chinese individuals.

\section{INTRODUCTION}

High-altitude pulmonary edema (HAPE), a lethal, non-cardiogenic form of pulmonary edema, is a severe form of acute mountain sickness occurring in susceptible individuals at altitudes $>2,500 \mathrm{~m}$ above sea level. It is an acute idiopathic disease that presents within 2 to 4 days after arrival at high altitude $[1,2]$ and can occur in two forms. The first form typically occurs in rapidly ascending un-acclimatized healthy lowlanders. The second form, also called re-entry HAPE, occurs in high-altitude dwellers who return from a sojourn at a low altitude. The two forms probably have the same pathophysiological basis
[3]. The incidence of HAPE is not definitively known, since it commonly occurs in remote mountainous regions, where data are often unavailable, and some patients may recover fully during descent [4]. Early symptoms of HAPE include cough, exertional dyspnea, and reduced exercise performance. As HAPE progresses, cough worsens and breathlessness at rest and sometimes orthopnea occur [3, 5]. It is generally known that high-altitude pulmonary edema tends to be associated with exaggerated pulmonary hypertension.

Since the response to the extreme high altitude environment varies among individuals and those who have previously experienced HAPE run a significant risk of 
Table 1: Main demographic characteristics of HAPE cases and controls

\begin{tabular}{|c|c|c|c|c|c|}
\hline & Cases & $\%$ & Control & $\%$ & $p^{\mathrm{a}}$ \\
\hline Total & 267 & & 304 & & \\
\hline \multicolumn{6}{|l|}{ Mean \pm SD } \\
\hline Age & $32.57 \pm 10.738$ & & $36.15 \pm 4.498$ & & $0.000^{*}$ \\
\hline Sex & & & & & $0.105^{\mathrm{b}}$ \\
\hline Female & 21 & 7.9 & 14 & 4.6 & \\
\hline Male & 246 & 92.1 & 290 & 95.4 & \\
\hline
\end{tabular}

$* p \leq 0.05$ indicates statistical significance.

a Independent samples t test.

b Two-sided $\chi 2$ tests.

Table 2: Candidate SNPs tested in the study

\begin{tabular}{lllccccc}
\hline SNP ID & Genes & Band & Role & Alleles A/B & $\boldsymbol{p}$-HWE & $\boldsymbol{p}^{\mathbf{a}}$ & OR(95 \% CI) \\
\hline rs275651 & $A G T R 1$ & $3 \mathrm{q} 24$ & Promoter & $\mathrm{A} / \mathrm{T}$ & 0.647 & $0.017^{*}$ & $0.65(0.45-0.93)$ \\
rs275652 & $A G T R 1$ & $3 \mathrm{q} 24$ & Promoter & $\mathrm{G} / \mathrm{T}$ & 0.647 & $0.016^{*}$ & $0.64(0.45-0.92)$ \\
rs2638360 & $A G T R 1$ & $3 \mathrm{q} 24$ & Intron & $\mathrm{G} / \mathrm{A}$ & 0.042 & 0.178 & $0.75(0.49-1.14)$ \\
rs4681443 & $A G T R 1$ & $3 \mathrm{q} 24$ & Intron & $\mathrm{A} / \mathrm{G}$ & 0.216 & 0.950 & $0.99(0.63-1.54)$ \\
rs1492099 & $A G T R 1$ & $3 \mathrm{q} 24$ & Intron & $\mathrm{G} / \mathrm{A}$ & 0.723 & 0.931 & $0.99(0.74-1.32)$ \\
rs1492097 & $A G T R 1$ & $3 \mathrm{q} 24$ & Intron & $\mathrm{A} / \mathrm{G}$ & 1.000 & 0.118 & $1.31(0.93-1.85)$ \\
rs4524238 & $A G T R 1$ & $3 \mathrm{q} 24$ & Intron & $\mathrm{A} / \mathrm{G}$ & 1.000 & 0.100 & $1.33(0.95-1.88)$ \\
\hline
\end{tabular}

SNP, single-nucleotide polymorphism; OR, odds ratio; 95\%CI, $95 \%$ confidence interval; HWE, Hardy-Weinberg equilibrium.

a Two-sided $\chi 2$ tests/Fisher's exact tests.

$* p \leq 0.05$ indicates statistical significance.

recurrence, it has been speculated that a constitutional or genetic component may underlie the development of this disease [6]. To date, a number of candidate genes, such as angiotensin-converting enzyme $(A C E)$, angiotensin $(A G T)$, renin $(R E N)$, aldosterone synthase (CYP11B2), and angiotensin II receptor type 1 (AGTR1), have been reported to be involved in the physiological response to hypoxic conditions. These genes have been associated with high risk of susceptibility to HAPE in some populations $[7,8]$.

As whole genome-wide association studies have not identified common variants in or near the AGTR1 gene associated with HAPE, the current study evaluated 7 SNPs that span the AGTR1 gene and their associations with HAPE risk in a case-control model comprising 571 individuals from a Han Chinese population.

\section{RESULTS}

A total of 267 patients with HAPE (aged 32.57 \pm 10.738 years) and 304 healthy controls (aged 36.15

\pm 4.498 years) were included in the current study.
Principle demographic characteristics of all participants are listed in Table 1 including age and sex. Table 2 lists the detailed information of candidate SNPs tested in the AGTR1 gene. We examined Hardy-Weinberg equilibrium in the controls, and all SNPs were found to be in Hardy-Weinberg equilibrium $(p \geq 0.05)$ except for the SNP rs2638360 ( $p=0.042)$. When comparing allelic frequency of HAPE cases and controls, we found two significant SNPs in the AGTR1 gene at a 5\% level $(\mathrm{rs} 275651, p=0.017$, odds ratio $[\mathrm{OR}]=0.65,95 \%$ confidence interval $[\mathrm{CI}]=0.45-0.93 ; \mathrm{rs} 275652, p=$ 0.016 , odds ratio $[\mathrm{OR}]=0.64,95 \%$ confidence interval $[\mathrm{CI}]=0.45-0.92)$.

The association analysis between the three SNPs (rs275651, rs275652 and rs4524238) and HAPE risk are summarized in Tables 3-5. Our results showed that two SNPs were significantly associated with susceptibility to HAPE in crude analysis by the heterozygote comparison (rs275651 AT vs. TT, OR $=0.55,95 \% \mathrm{CI}=0.36-0.83$; rs275652 GT vs. TT, OR $=0.55,95 \% \mathrm{CI}=0.36-0.83)$, dominant model analyses (rs275651, OR $=0.58,95 \%$ $\mathrm{CI}=0.39-0.86, p=0.007 ; \mathrm{rs} 275652, \mathrm{OR}=0.57,95 \%$ 
Table 3: Analysis of association between rs275651 polymorphism and risk of HAPE

\begin{tabular}{|c|c|c|c|c|c|c|c|}
\hline & \multirow{2}{*}{$\begin{array}{c}\text { Case (\%) } \\
N=266\end{array}$} & \multirow{2}{*}{$\begin{array}{c}\text { Control (\%) } \\
\mathrm{N}=304\end{array}$} & \multirow[t]{2}{*}{$p^{\mathrm{a}}$} & \multicolumn{2}{|l|}{ Crude } & \multicolumn{2}{|c|}{ Adjusted $^{\text {b }}$} \\
\hline & & & & OR $(95 \% \mathrm{CI})$ & $p$ & OR (95\%CI) & $p$ \\
\hline \multicolumn{8}{|l|}{ Genotype } \\
\hline $\mathrm{T} / \mathrm{T}$ & $218(82.0 \%)$ & $220(72.4 \%)$ & $0.017 *$ & 1 & $0.016^{*}$ & 1.00 & $0.018 *$ \\
\hline $\mathrm{A} / \mathrm{T}$ & $43(16.2 \%)$ & $79(26.0 \%)$ & & $0.55(0.36-0.83)$ & & $0.55(0.36-0.84)$ & \\
\hline $\mathrm{A} / \mathrm{A}$ & $5(1.8 \%)$ & $5(1.6 \%)$ & & $1.01(0.29-3.54)$ & & $1.05(0.29-3.82)$ & \\
\hline \multicolumn{8}{|l|}{ Dominant } \\
\hline $\mathrm{T} / \mathrm{T}$ & $218(81.9 \%)$ & $220(72.4 \%)$ & $0.007^{*}$ & 1 & $0.007 *$ & 1 & $0.008^{*}$ \\
\hline $\mathrm{A} / \mathrm{T}-\mathrm{A} / \mathrm{A}$ & $48(18.1 \%)$ & $84(27.6 \%)$ & & $0.58(0.39-0.86)$ & & $0.57(0.38-0.87)$ & \\
\hline \multicolumn{8}{|l|}{ Recessive } \\
\hline $\mathrm{T} / \mathrm{T}-\mathrm{A} / \mathrm{T}$ & $261(98.1 \%)$ & $299(98.4 \%)$ & 0.915 & 1 & 0.830 & 1 & 0.780 \\
\hline $\mathrm{A} / \mathrm{A}$ & $5(1.9 \%)$ & $5(1.6 \%)$ & & $1.15(0.33-4.00)$ & & $1.20(0.33-4.33)$ & \\
\hline \multicolumn{8}{|l|}{ Additive } \\
\hline $\mathrm{T} / \mathrm{T}$ & $218(82 \%)$ & $220(72.4 \%)$ & $0.018^{*}$ & $0.65(0.45-0.93)$ & $0.017^{*}$ & $0.65(0.45-0.94)$ & $0.021 *$ \\
\hline $\mathrm{A} / \mathrm{T}$ & $43(16.2 \%)$ & $79(26.0 \%)$ & & & & & \\
\hline $\mathrm{A} / \mathrm{A}$ & $5(1.8 \%)$ & $5(1.6 \%)$ & & & & & \\
\hline
\end{tabular}

a Two-sides $\chi 2$ test/Fisher's exact tests.

${ }^{\mathrm{b}}$ Adjusted for age and sex in a logistic regression model.

$* p \leq 0.05$ indicates statistical significance.

Table 4: Analysis of association between rs275652 polymorphism and risk of HAPE

\begin{tabular}{|c|c|c|c|c|c|c|c|}
\hline & \multirow{2}{*}{$\begin{array}{c}\text { Case (\%) } \\
N=267\end{array}$} & \multirow{2}{*}{$\begin{array}{c}\text { Control (\%) } \\
\mathbf{N}=\mathbf{3 0 4}\end{array}$} & \multirow[t]{2}{*}{$p^{\mathrm{a}}$} & \multicolumn{2}{|l|}{ Crude } & \multicolumn{2}{|c|}{ Adjusted $^{\mathrm{b}}$} \\
\hline & & & & OR $(95 \% C I)$ & $p$ & OR $(95 \% C I)$ & $p$ \\
\hline \multicolumn{8}{|l|}{ Genotype } \\
\hline $\mathrm{T} / \mathrm{T}$ & $219(82.0 \%)$ & $220(72.4 \%)$ & $0.016^{*}$ & 1 & $0.015^{*}$ & 1.00 & $0.017 *$ \\
\hline $\mathrm{G} / \mathrm{T}$ & $43(16.1 \%)$ & $79(26.0 \%)$ & & $0.55(0.36-0.83)$ & & $0.54(0.35-0.83)$ & \\
\hline $\mathrm{G} / \mathrm{G}$ & $5(1.9 \%)$ & $5(1.6 \%)$ & & $1.00(0.29-3.52)$ & & $1.05(0.29-3.81)$ & \\
\hline \multicolumn{8}{|l|}{ Dominant } \\
\hline $\mathrm{T} / \mathrm{T}$ & $219(82.0 \%)$ & $220(72.4 \%)$ & $0.006^{*}$ & 1 & $0.006^{*}$ & 1 & $0.007^{*}$ \\
\hline $\mathrm{G} / \mathrm{T}-\mathrm{G} / \mathrm{G}$ & $48(18.0 \%)$ & $84(27.6 \%)$ & & $0.57(0.38-0.86)$ & & $0.57(0.38-0.86)$ & \\
\hline \multicolumn{8}{|l|}{ Recessive } \\
\hline $\mathrm{T} / \mathrm{T}-\mathrm{G} / \mathrm{T}$ & $262(98.1 \%)$ & $299(98.4 \%)$ & 0.910 & 1 & 0.840 & 1 & 0.780 \\
\hline $\mathrm{G} / \mathrm{G}$ & $5(1.9 \%)$ & $5(1.6 \%)$ & & $1.14(0.33-3.99)$ & & $1.20(0.33-4.32)$ & \\
\hline \multicolumn{8}{|l|}{ Additive } \\
\hline $\mathrm{T} / \mathrm{T}$ & $219(82.0 \%)$ & $220(72.4 \%)$ & $0.017 *$ & $0.65(0.45-0.93)$ & $0.016^{*}$ & $0.65(0.45-0.94)$ & $0.020 *$ \\
\hline $\mathrm{G} / \mathrm{T}$ & $43(16.1 \%)$ & $79(26.0 \%)$ & & & & & \\
\hline $\mathrm{G} / \mathrm{G}$ & $5(1.9 \%)$ & $5(1.6 \%)$ & & & & & \\
\hline
\end{tabular}

a Two-sides $\chi 2$ test/Fisher's exact tests.

${ }^{\mathrm{b}}$ Adjusted for age and sex in a logistic regression model.

$* p \leq 0.05$ indicates statistical significance. 
Table 5: Analysis of association between rs4524238 polymorphism and risk of HAPE

\begin{tabular}{|c|c|c|c|c|c|c|c|}
\hline & \multirow{2}{*}{$\begin{array}{c}\text { Case (\%) } \\
N=267\end{array}$} & \multirow{2}{*}{$\begin{array}{c}\text { Control (\%) } \\
\mathrm{N}=304\end{array}$} & \multirow[t]{2}{*}{$p^{\mathrm{a}}$} & \multicolumn{2}{|l|}{ Crude } & \multicolumn{2}{|c|}{ Adjusted $^{\mathrm{b}}$} \\
\hline & & & & OR (95\%CI) & $p$ & OR $(95 \% \mathrm{CI})$ & $p$ \\
\hline \multicolumn{8}{|l|}{ Genotype } \\
\hline $\mathrm{G} / \mathrm{G}$ & $196(73.4 \%)$ & $237(78.0 \%)$ & 0.180 & 1.00 & 0.180 & 1.00 & 0.082 \\
\hline $\mathrm{A} / \mathrm{G}$ & $62(23.2 \%)$ & $63(20.7 \%)$ & & $1.19(0.80-1.77)$ & & $1.30(0.86-1.97)$ & \\
\hline $\mathrm{A} / \mathrm{A}$ & $9(3.4 \%)$ & $4(1.3 \%)$ & & $2.72(0.83-8.97)$ & & $3.20(0.94-10.90)$ & \\
\hline \multicolumn{8}{|l|}{ Dominant } \\
\hline $\mathrm{G} / \mathrm{G}$ & $196(73.4 \%)$ & $237(78.0 \%)$ & 0.205 & 1 & 0.210 & 1 & 0.088 \\
\hline $\mathrm{A} / \mathrm{G}-\mathrm{A} / \mathrm{A}$ & $71(26.6 \%)$ & $67(22.0 \%)$ & & $1.28(0.87-1.88)$ & & $1.41(0.95-2.10)$ & \\
\hline \multicolumn{8}{|l|}{ Recessive } \\
\hline G/G-A/G & $258(96.6 \%)$ & $300(98.7 \%)$ & 0.100 & 1 & 0.098 & 1 & 0.065 \\
\hline $\mathrm{A} / \mathrm{A}$ & $9(3.4 \%)$ & $4(1.3 \%)$ & & $2.62(0.80-8.60)$ & & $3.01(0.89-10.18)$ & \\
\hline \multicolumn{8}{|l|}{ Additive } \\
\hline $\mathrm{G} / \mathrm{G}$ & $196(73.4 \%)$ & $237(78.0 \%)$ & 0.108 & $1.32(0.94-1.84)$ & 0.110 & $1.44(1.01-2.04)$ & $0.040 *$ \\
\hline $\mathrm{A} / \mathrm{G}$ & $62(23.2 \%)$ & $63(20.7 \%)$ & & & & & \\
\hline $\mathrm{A} / \mathrm{A}$ & $9(3.4 \%)$ & $4(1.3 \%)$ & & & & & \\
\hline
\end{tabular}

a Two-sides $\chi 2$ test/Fisher's exact tests.

${ }^{\mathrm{b}}$ Adjusted for age and sex in a logistic regression model.

$* p \leq 0.05$ indicates statistical significance.

$\mathrm{CI}=0.38-0.86, p=0.006$ ), and additive model analyses $(\mathrm{rs} 275651, \mathrm{OR}=0.65,95 \% \mathrm{CI}=0.45-0.93, p=0.017$; rs $275652, \mathrm{OR}=0.65,95 \% \mathrm{CI}=0.45-0.93, p=0.016$ ). After adjustment for age and sex, these associations remained significant $(p<0.05)$. We also observed another susceptibility SNP, rs 4524238 , by additive model analyses (adjusted OR $=1.44,95 \% \mathrm{CI}=1.01-2.04, p=0.040$ ) after adjusting for age and sex.

Two haplotype blocks were detected when using the $\mathrm{D}^{\prime}$ measure of linkage disequilibrium (Figure 1). One haplotype block spanned rs275651 and rs275652 in promoter region (Haploblock1) and another haplotype block contained rs1492097 and rs4524238 in intron region (Haploblock2). The relationship between AGTR1 haplotype and the risk of the occurrence of HAPE were listed in Table 6 . Haplotype "AG" in Block 1 has been shown to be significantly associated with the risk of HAPE in crude analysis $(\mathrm{OR}=0.65,95 \% \mathrm{CI}=0.45$ $0.93, p=0.018)$, and the result remained significant after adjustment for age and sex $(p<0.05)$. In Block 2 , a significant increase risk between the haplotype "AA" and susceptibility of HAPE was only observed after adjustment for age and sex (adjusted OR $=1.44,95 \% \mathrm{CI}$ $=1.02-2.05, p=0.040$ ).

\section{DISCUSSION}

In this case-control study, we examined the association between polymorphisms in the AGTR1 gene and propensity to develop HAPE. Two susceptibility loci (rs275651 and rs275652) were associated with decreased risk of HAPE (rs275651, OR $=0.65$; rs275652, OR = 0.64 ), whereas rs 4524238 was associated with significantly increased risk $(\mathrm{OR}=1.44)$. Additionally, haplotype-based tests of association indicated a risk effect of up to $44 \%$ for "AA" haplotype and a protective effect of up to $35 \%$ for "AG" haplotype. To our knowledge, this is the first study to report the effect of these variants in HAPE individuals.

The AGTRl gene, which maps to chromosome $3 \mathrm{q} 21-\mathrm{q} 25$ and consists of five exons and four introns spanning approximately $45.1 \mathrm{~kb}$ of genomic DNA, encodes the type 1 angiotensin II receptor $\left(\mathrm{AT}_{1} \mathrm{R}\right)$. $\mathrm{AT}_{1} \mathrm{R}$ is a 7 transmembrane domain G-protein coupled receptor, widely expressed in different tissues and known to mediate most of the classical actions of Angiotensin II, including vasoconstriction and cellular growth and proliferation [9, 10] [11]. In hypoxic conditions Angiotensin II interacts with $\mathrm{AT}_{1} \mathrm{R}$ to modulate the pulmonary vasoconstrictive response [12]. 
We speculate that the AGTR1 gene is critical in HAPE pathogenesis its association with HAPE has also been demonstrated in a Japanese population [8]. AGTR1 gene polymorphisms are frequently observed [13], and a number of different polymorphisms in the AGTR1 gene have been identified [14]. However, variants of the angiotensin receptor AGTR1 were not found to associate with AMS and HAPE in various ethnic groups in earlier studies $[15,16]$. In the current study, seven SNPs (rs275651, rs275652, rs2638360, rs4681443, rs 1492099, rs1492097, rs4524238) were genotyped; of these rs275651, rs275652 and rs4524238 were related to HAPE susceptibility. Carriers of the rs275651 "A" allele exhibited a statistically significant decrease of $43 \%$ and $35 \%$ in HAPE susceptibility by the dominant model and additive model, respectively. rs 275652 also decreased risk by $43 \%$ and $35 \%$. In haplotype analysis, rs275651 and rs275652 showed a strong linkage disequilibrium pattern and formed one haplotype block. These results indicate that these two variants conferred a possible protective effect against HAPE. Additionally, an additive effect of the rs4524238 "A" allele was observed, resulting in a multiple increased 1.44-fold risk of HAPE. This study is the first to report the rs4524238 variant in any ethnic group and our findings suggest that this variant significantly contributes to the development of HAPE. As the rs4524238 polymorphism is an intronic mutant, the mechanism by which it confers risk is unclear. It is possible that the variant affects the transcription of $A G T R 1$ or is in linkage disequilibrium with true functional variations directly involved in HAPE susceptibility.

Several potential limitations should be taken into consideration. First, participants in this study were limited to the Han Chinese population; whether the results are also applicable to other ethnic groups is currently unknown. Second, the limited sample size of our study did not allow subgroup analysis. Third, when multiple comparisons were carried out, we did not apply Bonferroni correction to control potential type I errors because this correction method might have increased the chance of a type II error. Finally, additional studies including fine mapping and functional validation are needed to clarify these findings and the mechanisms by which this polymorphism increases HAPE risk.

To sum up, we identified the first significant associations between SNPs rs275651, rs275652 and rs4524238 and HAPE susceptibility, which may provide important biological markers for early diagnosis and prognosis for HAPE patients.

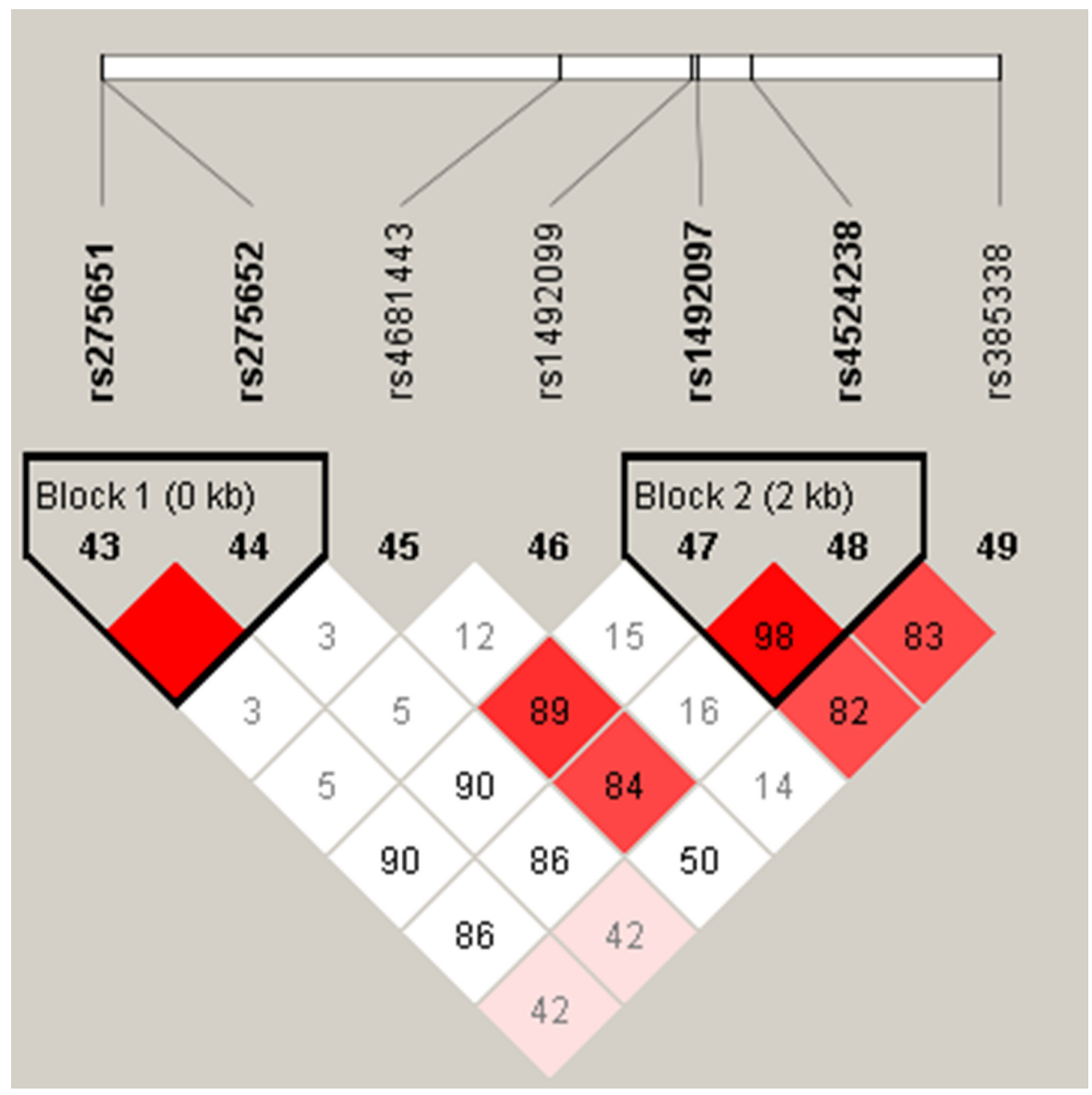

Figure 1: Haplotype block map for all the SNPs of the AGTR1 gene. 
Table 6: AGTR1 haplotype frequencies and the association with HAPE risk in case and control subjects

\begin{tabular}{|c|c|c|c|c|c|c|c|c|}
\hline \multirow{2}{*}{ Block } & \multirow{2}{*}{ Haplotype } & \multirow{2}{*}{$\begin{array}{l}\text { Freq } \\
\text { (case) }\end{array}$} & \multirow{2}{*}{ Freq (control) } & \multirow{2}{*}{$p^{\mathbf{a}}$} & \multicolumn{2}{|c|}{ Crude } & \multicolumn{2}{|c|}{ Adjusted $^{b}$} \\
\hline & & & & & OR $(95 \% C I)$ & $p$ & OR $(95 \% C I)$ & $p$ \\
\hline \multirow[t]{2}{*}{1} & $\mathrm{TT}$ & 0.901 & 0.854 & $0.016 *$ & 1 & & 1 & \\
\hline & $\mathrm{AG}$ & 0.099 & 0.146 & $0.016^{*}$ & $\begin{array}{c}0.65(0.45- \\
0.93)\end{array}$ & $0.018 *$ & $\begin{array}{c}0.65(0.45- \\
0.94)\end{array}$ & $0.022 *$ \\
\hline \multirow[t]{2}{*}{2} & GG & 0.850 & 0.881 & 0.124 & 1 & & 1 & \\
\hline & $\mathrm{AA}$ & 0.150 & 0.116 & 0.087 & $\begin{array}{c}1.32(0.94- \\
1.85)\end{array}$ & 0.110 & $\begin{array}{c}1.44(1.02- \\
2.05)\end{array}$ & $0.040 *$ \\
\hline
\end{tabular}

a Two-sides $\chi 2$ test/Fisher's exact tests.

${ }^{\mathrm{b}}$ Adjusted for age and sex in a logistic regression model.

$* p \leq 0.05$ indicates statistical significance.

\section{MATERIALS AND METHODS}

\section{Study participants}

From March 2013 to October 2015, we consecutively recruited 267 HAPE patients (HAPEsusceptible cohorts) and 304 ethnically matched control subjects (HAPE-resistant cohorts) from the General Hospital of Tibet Military Region, Lhasa, China. All of the subjects were of genetically unrelated Chinese Han ancestry and informed consent was obtained from each individual enrolled. The patient was immediately admitted to the hospital if anoxic symptoms arose at high altitude. All HAPE patients were diagnosed and confirmed to suffer from HAPE following previously described criteria [17]. A standardized questionnaire and clinical examination were used to exclude any previous history of cardiopulmonary disease. Patients had not received any drug treatment before blood samples were taken. They were all in good health after timely treatment. The control subjects were defined as HAPE- resistant cohorts due to their resistance to HAPE during exposure to similar high-altitude environment. No subjects reported any history of HAPE or other cardiopulmonary disorders in a questionnaire sheet that contained the components of Lake Louise Score [18]. Blood samples were taken from the study participants, and immediately frozen and stored at $-70 \mathrm{C}$ until analysis. The study was approved by the Human Research Committee for Approval of Research Involving Human Subjects.

\section{SNP selection and genotyping}

SNPs within the AGTR1 gene with minor allele frequency $(\mathrm{MAF})>0.05$ in the HapMap Asian population were obtained from PubMed databases and single-nucleotide polymorphism (SNP) databases [the dbSNP (NCBI) and the Japanese SNP database (JSNP)]. DNA was extracted from peripheral whole blood cells by the GoldMag-Mini Whole Blood Genomic DNA Purification Kit (GoldMag Co. Ltd. Xian, China) following the manufacturer's protocols, and DNA concentration of each sample was determined by spectrometry (DU530 UV/VIS spectrophotometer, Beckman Instruments, Fullerton, CA, USA). The PCR and extension primers were designed using the MassARRAY Assay Design 3.0 software (Sequenom, Inc.). SNPs were genotyped with the Sequenom MassARRAY RS1000 according to the instructions of the manufacturer [19]. We performed data management and analyses using Sequenom MassArray TYPER 4.0 software.

\section{Statistical analysis}

Statistical analysis was performed using Microsoft Excel and SPSS 16.0(SPSS, Chicago, IL, USA). All hypothesis testing was two-sided with a $p$ value of 0.05 deemed as significant. Observed genotype frequencies for $A G T R 1$ polymorphisms in controls were tested for deviation from Hardy-Weinberg equilibrium (HWE) using a goodness-of-fit $\chi 2$ test. Odds ratios (ORs) and $95 \%$ confidence intervals (CI) were estimated by unconditional logistic regression after adjustment for age and sex. The genotypic associations of every polymorphism were compared under the dominant, recessive, and additive genetic models as suggested by previous studies using the same method [20, 21]. Finally, the Haploview software package (http:// www.broadinstitute.org/haploview) and the SHEsis software platform (http://analysis.bio-X.cn/myAnalysis. php) was used to analyze the linkage disequilibrium (LD) structure, calculate $\mathrm{D}^{\prime}$ to define haplotype blocks and estimate haplotype frequencies $[22,23]$. 


\section{ACKNOWLEDGMENTS}

This work is supported by National Natural Science Foundations (No. 81560516), Major science and technology research projects of Xizang (Tibet) Autonomous Region (2015 and 2016), and the 2016 Young University Teacher Innovative Support Program of Xizang (Tibet) Autonomous Region (QCZ2016-38).

\section{CONFLICTS OF INTEREST}

The authors have no conflicts of interest to report.

\section{REFERENCES}

1. Bhagi S, Srivastava S, Singh SB. High-altitude pulmonary edema: review. Journal of occupational health. 2014; 56 : 235-243.

2. Luo Y, Zou Y, Gao Y. Gene polymorphisms and highaltitude pulmonary edema susceptibility: a 2011 update. Respiration. 2012; 84: 155-162.

3. Paralikar SJ. High altitude pulmonary edema-clinical features, pathophysiology, prevention and treatment. Indian journal of occupational and environmental medicine. 2012; 16: 59-62.

4. Hall DP, Duncan K, Baillie JK. High altitude pulmonary oedema. Journal of the Royal Army Medical Corps. 2011; 157: 68-72.

5. Bartsch P, Mairbaurl H, Swenson ER, Maggiorini M. High altitude pulmonary oedema. Swiss medical weekly. 2003; 133: $377-384$.

6. Mortimer H, Patel S, Peacock AJ. The genetic basis of highaltitude pulmonary oedema. Pharmacology \& therapeutics. 2004; 101: 183-192.

7. Qi Y, Niu W, Zhu T, Zhou W, Qiu C. Synergistic effect of the genetic polymorphisms of the renin-angiotensinaldosterone system on high-altitude pulmonary edema: a study from Qinghai-Tibet altitude. European journal of epidemiology. 2008; 23: 143-152.

8. Hotta J, Hanaoka M, Droma Y, Katsuyama Y, Ota M, Kobayashi T. Polymorphisms of renin-angiotensin system genes with high-altitude pulmonary edema in Japanese subjects. Chest. 2004; 126: 825-830.

9. Su S, Chen J, Zhao J, Huang J, Wang X, Chen R, Gu D. Angiotensin II type I receptor gene and myocardial infarction: tagging SNPs and haplotype based association study. The Beijing atherosclerosis study. Pharmacogenetics. 2004; 14: 673-681.

10. Plummer S, Tower C, Alonso P, Morgan L, Baker P, Broughton-Pipkin F, Kalsheker N. Haplotypes of the angiotensin II receptor genes AGTR1 and AGTR2 in women with normotensive pregnancy and women with preeclampsia. Human mutation. 2004; 24: 14-20.

11. Davies E, Bonnardeaux A, Plouin PF, Corvol P, Clauser E. Somatic mutations of the angiotensin II (AT1) receptor gene are not present in aldosterone-producing adenoma. The Journal of clinical endocrinology and metabolism. 1997; 82: 611-615.

12. Morrell NW, Morris KG, Stenmark KR. Role of angiotensin-converting enzyme and angiotensin II in development of hypoxic pulmonary hypertension. The American journal of physiology. 1995; 269: H1186-1194.

13. Baudin B. Polymorphism in angiotensin II receptor genes and hypertension. Experimental physiology. 2005; 90: 277-282.

14. Bonnardeaux A, Davies E, Jeunemaitre X, Fery I, Charru A, Clauser E, Tiret L, Cambien F, Corvol P, Soubrier F. Angiotensin II type 1 receptor gene polymorphisms in human essential hypertension. Hypertension. 1994; 24: 63-69.

15. Koehle MS, Wang P, Guenette JA, Rupert JL. No association between variants in the ACE and angiotensin II receptor 1 genes and acute mountain sickness in Nepalese pilgrims to the Janai Purnima Festival at $4380 \mathrm{~m}$. High altitude medicine \& biology. 2006; 7: 281-289.

16. Alvarez R, Terrados N, Ortolano R, Iglesias-Cubero G, Reguero JR, Batalla A, Cortina A, Fernandez-Garcia B, Rodriguez C, Braga S, Alvarez V, Coto E. Genetic variation in the renin-angiotensin system and athletic performance. European journal of applied physiology. 2000; 82: 117-120.

17. Kobayashi T, Koyama S, Kubo K, Fukushima M, Kusama S. Clinical features of patients with high-altitude pulmonary edema in Japan. Chest. 1987; 92: 814-821.

18. Sikri G, Dua S. High altitude pulmonary edema and hypoxia-related genes - Genet. Mol. Res. 14 (3): 11562-11572 "Correlation between single nucleotide polymorphisms in hypoxia-related genes and susceptibility to acute high-altitude pulmonary edema". Genetics and molecular research: GMR. 2015; 14: 15904-15905.

19. Gabriel S, Ziaugra L, Tabbaa D. SNP genotyping using the Sequenom MassARRAY iPLEX platform. Current protocols in human genetics / editorial board, Jonathan L Haines [et al]. 2009; Chapter 2: Unit 212.

20. Niu D, Ren Y, Xie L, Sun J, Lu W, Hao Y, Zhang Y, Yin A, Li H, Lv J, Li S. Association between CCDC132, FDX1 and TNFSF 13 gene polymorphisms and the risk of $\operatorname{IgA}$ nephropathy. Nephrology. 2015; 20: 908-915.

21. Jin TB, Ren Y, Shi X, Jiri M, He N, Feng T, Yuan D, Kang L. Genetic variations in the CLNK gene and ZNF518B gene are associated with gout in case-control sample sets. Rheumatology international. 2015; 35: 1141-1147.

22. Barrett JC, Fry B, Maller J, Daly MJ. Haploview: analysis and visualization of LD and haplotype maps. Bioinformatics (Oxford, England). 2005; 21: 263-265.

23. Shi YY, He L. SHEsis, a powerful software platform for analyses of linkage disequilibrium, haplotype construction, and genetic association at polymorphism loci. Cell research. 2005; 15: 97-98. 